Pacific Journal of Mathematic 


\title{
LOCALIZATION IN FULLY BOUNDED NOETHERIAN RINGS
}

\author{
BRUNO J. MÜLleR
}

The paper defines and studies links between the prime ideals of a noncommutative fully bounded noetherian ring, and their role as obstructions to localizability: a localization with properties similar to those of the localization of a commutative ring at a prime ideal, can be constructed if and only if the equivalence class determined by the links is finite. For rings with polynomial identity, the links are described in more detail via an inductive procedure over the PI-degree, and several examples are constructed.

I. Preliminaries. The attempt to localize a noncommutative noetherian ring at its prime ideals leads to the study of classical sets of prime ideals (i.e. finite incomparable sets $\left\{P_{1}, \cdots, P_{n}\right\}$ such that the associated torsion theory has the Ore condition and the Artin Rees property) and in particular of clans (i.e., minimal classical sets). It was shown in [16] that a prime ideal belongs to at most one clan, and that the existence of enough clans (i.e. each prime ideals belongs to a clan) amounts to localizability at all prime ideals; cf. this paper for more detail.

The very existence of nontrivial clans (i.e., clans with more than one member) is evidence of the presence of links between prime ideals which constitute obstructions to localizability. The purpose of this paper is to define and study these links explicitely.

To do so, we restrict attention to FBN-rings (right- and leftfully bounded noetherian rings), where we have these fundamental results of [9] on Krull dimension $\kappa$ available: Every uniform module is $\alpha$-smooth for some ordinal $\alpha$, i.e., all nonzero submodules have the same Krull dimension $\alpha$. Every finitely generated $\alpha$-smooth module has an (essentially unique) $\alpha$-composition series (called basic series in [9]). The $\alpha$-composition factors, also called $\alpha$-critical modules, are characterized as the uniform nonsingular $R / P$-modules, for the various prime ideals $P$ of the ring $R$ with $\kappa(R / P)=\alpha$. Any $R-R$ bimodule which is finitely generated on both sides, has the same Krull dimension on both sides.

The FBN-assumption is natural for other reasons: It makes the Gabriel correspondence between spec $R$ and the collection of indecomposable injective modules one-to-one, via $E(R / P)=E_{P}^{n p}$; i.e., 
prime ideals "separate modules" [11]. It ensures that any localizable semiprime ideal is automatically classical (combine [9], 3.6 with [8], 4.4(5)). Recall also that primitive ideals of FBN-rings are maximal with simple artinian factorrings, and that noetherian PI-rings (i.e. noetherian rings with polynomial identity) are FBN.

A useful criterion from [8]: A semiprime ideal $S$ of a rightnoetherian ring $R$ is right-localizable (i.e., the multiplicative set $\mathscr{C}(S)$ has the right-Ore condition) if and only if $\mathscr{C}(S)$ operates regularly on the injective hull $E(R / S)$.

Some terminology: module means right-module; but ideal, noetherian, Ore etc. means left- and right-ideal, -noetherian, -Ore etc., unless specified otherwise. $\kappa(M)$ is the Krull dimension [5], and $E(M)$ is the injective hull, of the module $M . J(R)$ is the Jacobson radical, and $\mathscr{C}(S)$ is the multiplicative set of modulo $S$ regular elements, of a ring $R$. The collection of prime ideals $P$ of an FBN-ring $R$ with $\kappa(R / P)=\alpha$ will be called the $\alpha$-stratum of spec $R$ : note that distinct prime ideals in the same stratum are incomparable ([5], 7.2).

\section{FBN-rings.}

Lemma 1. Let $R$ be an FBN-ring, $P$ and $Q$ prime ideals in the $\alpha$-stratum, and $\bar{R}=R / Q P$. Then $\mathscr{C}(\bar{Q} \cap \bar{P})$ is Ore in $\bar{R}$, and the corresponding quotient ring $A$ is artinian, with two maximal ideals $P A$ and $Q A$ and $J(A)^{2}=0$.

Proof (cf. [9], proof of 5.2). Let $E=E\left(R /(Q \cap P)_{R}\right)$ which is $\alpha$-smooth, and $\left.F=\operatorname{ann}_{E}(Q P)=E(\bar{R} / \bar{Q} \cap \bar{P})_{\bar{R}}\right) . \quad \mathscr{C}(\bar{Q} \cap \bar{P})$ operates regularly on $F$ : if $0 \neq x \in F$ and $c \in \mathscr{C}(Q \cap P)$, let $\overline{x R}$ be the top factor of an $\alpha$-composition series of $x R$; then $\operatorname{ann}_{R}(\overline{x R})=P^{\prime}$ is a prime ideal with $\kappa\left(R / P^{\prime}\right)=\alpha . \quad F Q P=0$ implies $Q P \subset P^{\prime}$ hence $Q$ or $P \subset P^{\prime}$ hence $Q$ or $P=P^{\prime}$ since all three lie in the $\alpha$-stratum; hence $c \in \mathscr{C}(Q \cap P)=\mathscr{C}(Q) \cap \mathscr{C}(P) \subset \mathscr{C}\left(P^{\prime}\right)$. Thus $c$ operates regularly on the non-singular $R / P^{\prime}$-module $\overline{x R}$, hence $x c \neq 0$.

By the criterion cited in the preliminaries, $\mathscr{C}(\bar{Q} \cap \bar{P})$ is an Oreset. The quotient ring $A$ is noetherian, semilocal with maximal ideals $P A$ and $Q A$ and $J(A)=(Q \cap P) A$, hence $J(A)^{2}=0$ since $(Q \cap P)^{2} \subset Q P$; hence $A$ is artinian.

REMARK. The kernel of $\bar{R} \rightarrow \bar{A}$, i.e. the torsion radical of $\bar{R}$ for the $(\bar{Q} \cap \bar{P})$-torsion theory, is $\operatorname{ann}_{\bar{R}}(F)=\operatorname{ann}_{\bar{R}} \operatorname{ann}_{E}(Q P)=$ 
$\operatorname{ann}_{R} \operatorname{ann}_{E}(Q P) / Q P$, since $F$ is an injective cogenerator for $A$.

Proposition 2. In the situation of Lemma 1, the following are equivalent:

(1) there is a short exact sequence $0 \rightarrow X \rightarrow Y \rightarrow Z \rightarrow 0$ of $R$ modules such that $Y$ is uniform, $X$ and $Z$ are critical, and $\operatorname{ann}_{R}(X)=P, \operatorname{ann}_{R}(Z)=Q$;

(2) $\operatorname{Ext}_{A}^{1}(A / Q A, A / P A) \neq 0$;

(3) $\operatorname{Tor}_{1}^{A}(A / Q A, A / P A) \neq 0$;

(4) $(Q \cap P) I \subset Q P$ implies $I \subset P$, for every ideal $I$ of $R$.

Definition. If the conditions of Proposition 2 hold, we say that a (right-) link exists from $P$ to $Q$, and write $P \leadsto Q$.

REMARK. Since (3) is left-right-symmetric (as $I A=A I$ for every ideal $I$ of $R$ ), these conditions are also equivalent to their left-rightanalogues with the roles of $P$ and $Q$ interchanged, i.e., to the existence of a left-link from $Q$ to $P$.

Proof. If $0 \rightarrow X \rightarrow Y \rightarrow Z \rightarrow 0$ is given as in (1), then up to isomorphism $X \subset Y \subset E_{P}$ since $X$ is a uniform nonsingular $R / P$-module and since $Y$ is uniform; similarly $Z \subset E_{Q}$. Under localization, $0 \rightarrow X \otimes A \rightarrow Y \otimes A \rightarrow Z \otimes A \rightarrow 0$ stays exact, $X \otimes A$ and $Z \otimes A$ are simple $A$-modules of type $A / P A$ and $A / Q A$ respectively, and the sequence is nonsplit since $Y \otimes A$ stays uniform. Hence $\operatorname{Ext}_{A}^{1}(A / Q A, A / P A) \neq 0$, i.e., (2).

Conversely if (2) holds, then there is a nonsplit exact sequence $0 \rightarrow X \rightarrow Y \rightarrow Z \rightarrow 0$ of $A$-modules, where $X$ and $Z$ are simple with annihilators $P A$ and $Q A$ respectively. Nonsplitting forces $Y$ to be uniform as $A$-module, hence $X, Y$, and $Z$ are uniform as $R$-modules; and the annihilators of $\mathrm{X}$ and $Z$ in $R$ are just $P$ and $Q$, since these prime ideals are closed in the $(Q \cap P)$-torsion theory. Moreover $X$ and $Z$ are $(Q \cap P)$-torsionfree hence nonsingular $R / P$ - respectively $R / Q$-modules, hence critical; and we have recovered the situation (1).

The equivalence of (2) and (3) follows from the homological duality isomorphism ([1], 120), using that $\operatorname{hom}_{A / P A}(-, A / P A)$ is a duality between finitely generated right- and left-modules over the simple artinian ring $A / P A$, carrying the right-module $A / P A$ into the left-module $A / P A$.

One easily computes $\operatorname{Tor}_{1}^{A}(A / Q A, A / P A)=(Q A \cap P A) / Q P A=$ $(Q \cap P) A$, hence $\operatorname{Tor}_{1}^{A}(A / Q A, A / P A)=0$ if and only if there is $c \in \mathscr{C}(Q \cap P)$ with $(\bar{Q} \cap \bar{P}) c=0$ in $\bar{R}=R / Q P$. But this is equivalent 
to the existence of an ideal $I \not \subset P$ with $(Q \cap P) I \subset Q P$ (take $I=R c R+P$ and use the Lemma in [7]), establishing the equivalence of (3) and (4).

REMARKs. (1) One can deduce that the existence of a self-link from $P$ to $P$ is equivalent to $P \supsetneqq P^{(2)}$, the symbolic square of $P$ [13]. Analogously the existence of a link from $P$ to $Q$ may be interpreted to mean that $Q \cap P$ is strictly larger than an appropriately defined symbolic product.

(2) If $P$ and $Q$ are maximal ideals (i.e., in the $O$-stratum), then $A=R / Q P$ (since the latter is already artinian), and a link $P m Q$ exists if and only if $Q \cap P \supsetneqq Q P$.

(3) The links between finitely many prime ideals $P_{1}, \cdots, P_{n}$ (in the same stratum of spec $R$ ) can be visualized in an artinian ring: the proofs of Lemma 1 and Proposition 2 go through for $\bar{R}=R / S^{2}$ where $S=P_{1} \cap \cdots \cap P_{n}$, and its artinian quotient ring $A$ with maximal ideals $P_{i} A$ and $J(A)^{2}=0$. Hence a link $P_{i} m P_{j}$ exists in $R$ if and only if $P_{j} A \cap P_{i} A \supsetneqq P_{j} P_{i} A$.

The structure of any artinian ring $A$ with $J(A)^{2}=0$, is given by a finite family of primary (hence local up to Morita equivalence) artinian rings $\left(A_{i}, M_{i}\right)$ with $M_{i}^{2}=0$ and of bimodules $X_{i j}(i \neq j)$ over the simple artinian rings $A_{i} / M_{i}$ and $A_{j} / M_{j}$, with zero-multiplication $X_{i j} X_{j k}=0$ [14]. In such a representation a link $P_{i} m P_{j}$ exists if and only if $X_{j i} \neq 0$. Since for suitable $A_{i}$ the $X_{i j}$ can be chosen zero or nonzero at will ([15], Lemma 1), any finite directed graph can be realized as the graph of links between the prime ideals of an artinian ring.

Note on the other hand that for a bounded HNP-ring, the occuring graphs are very restricted: they are directed circuits, corresponding to the cycles of [3].

Lemma 3. The following are equivalent for prime ideals $P, Q$ in the $\alpha$-stratum of an FBN-ring $R$ :

(1) there is a nonzero homomorphism $\phi: E_{P} \rightarrow E_{Q}$;

(2) there exists $e \in E_{P}$ with $\operatorname{ann}_{R}(e R) \subset Q$;

(3) $Q$ is the annihilator of an $\alpha$-composition factor of $E_{P}$.

Proof. If $0 \neq \phi: \quad E_{P} \rightarrow E_{Q}$ is given, then there is $e \in E_{P}$ with $\phi(e) \neq 0$ and $\operatorname{ann}_{R}(\dot{\phi}(e) R)=Q$. Then $\dot{\phi}(e)$ ann $(e R)=0$ hence ann $(e R) \subset Q$. 
If $e \in E_{P}$ with ann $(e R) \subset Q$ is given, consider an $\alpha$-composition series $0=B_{0} \varsubsetneqq \cdots \varsubsetneqq B_{n}=e R$; then the $\operatorname{ann}_{R}\left(B_{i} / B_{i-1}\right)=P_{i}$ are prime ideals of $R$ with $\kappa\left(R / P_{i}\right)=\alpha$. Then $e R \cdot P_{n} \cdots P_{1}=0$ hence $P_{n} \cdots P_{1} \subset$ ann $(e R) \subset Q$ hence $P_{i} \subset Q$ for some $i$.

If finally $Q$ is the annihilator of the $\alpha$-composition factor $A / B$ of $E_{P}$, then $A / B$ is a uniform nonsingular $R / Q$-module hence embedds into $E_{Q}$, and the natural map $A \rightarrow A / B$ extends to a nonzero homomorphism $E_{P} \rightarrow E_{Q}$.

Proposition 4. Let $P, Q$ be distinct prime ideals in the $\alpha$-stratum of an FBN-ring $R$. Then a finite chain of links $P m P_{1} m \cdots m Q$ exists if and only if there is a finite chain of nonzero homomorphisms $E_{P} \rightarrow E_{P^{\prime}} \rightarrow \cdots \rightarrow E_{Q}$.

Proof. If $P \leadsto Q$ is given, one has $0 \rightarrow X \rightarrow Y \rightarrow Z \rightarrow 0$ with $X \subset Y \subset E_{P}$ and $Z \subset E_{Q}$ as observed in the proof of Proposition 2, and $Y \rightarrow Z$ extends to a nonzero homomorphism $E_{P} \rightarrow E_{Q}$.

Conversely, associate with every $Q^{\prime}$ in the $\alpha$-stratum, for which a nonzero homomorphism $E_{P} \rightarrow E_{Q^{\prime}}$ exists, an $\alpha$-composition series of shortest length for a finitely generated submodule of $E_{P}$, for which $Q^{\prime}$ is the annihilator of a factor (necessarily the top factor); this is possible by Lemma 3 . We proceed by induction over this shortest length.

Thus let $0=B_{0} \varsubsetneqq \cdots \varsubsetneqq B_{n-2} \varsubsetneqq B_{n-1} \varsubsetneqq B_{n}$ be a $\alpha$-composition series of shortest length in $E_{P}$ with ann $\left(B_{n} / B_{n-1}\right)=Q$. The inclusion $B_{n-1} / B_{n-2} \subset B_{n} / B_{n-2}$ is essential, since otherwise

$$
B_{n-1} / B_{n-2} \oplus B / B_{n-2} \subset B_{n} / B_{n-2}
$$

hence $B / B_{n-2} \neq 0$ embedds into $B_{n} / B_{n-1}$ hence is critical with annihilator $Q$, producing an $\alpha$-composition series $0=B_{0} \varsubsetneqq \cdots \varsubsetneqq B_{n-2} \varsubsetneqq B$ of shorter length for $Q$, a contradiction. Thus

$$
E\left(B_{n} / B_{n-2}\right)=E\left(B_{n-1} / B_{n-2}\right)=E_{Q^{\prime}}
$$

where $Q^{\prime}=\operatorname{ann}\left(B_{n-1} / B_{n-2}\right)$ has an $\alpha$-composition series

$$
0=B_{0} \varsubsetneqq \cdots \varsubsetneqq B_{n-2} \subsetneq B_{n-1}
$$

of shorter length hence a finite chain $P \leadsto P_{1} \cdots m Q^{\prime}$ by induction. Moreover a link $Q^{\prime} \leadsto Q$ exists from the exact sequence

$$
0 \longrightarrow B_{n-1} / B_{n-2} \longrightarrow B_{n} / B_{n-2} \longrightarrow B_{n} / B_{n-1} \longrightarrow 0
$$


and Proposition 2 (4), and we are done.

REMARK. The proceeding proof motivates the notation $P \leadsto m Q$, if $\kappa(R / P)=\kappa(R / Q)$ and if a nonzero homomorphism $E_{P} \rightarrow E_{Q}$ exists. Note that all the intermediate primes in both chains of Proposition 4 belong automatically to the $\alpha$-stratum.

By Proposition 4, $P \leadsto Q$ and $P \leadsto m Q$ generate the same equivalence relation on spec $R$. By the remark following Proposition 2 , the very same equivalence relation arises from left-links. The equivalence class of $P$ in this equivalence relation will be written class $(P)$.

Theorem 5. A prime ideal $P$ of an FBN-ring $R$ belongs to $a$ clan if and only if class $(P)$ is finite; then clan $(P)=\operatorname{class}(P)$.

Proof. Assume class $(P)$ to be finite, and put $S=\bigcap$ class $(P)$. If $S$ is not right-localizable, there exists $c \in \mathscr{C}(S)$ and $0 \neq e \in E_{Q}$ for some $Q \in \operatorname{class}(P)$ with $e c=0$, by Jategaonkar's criterion. If the top factor $\overline{e R}$ of an $\alpha$-composition series of $e R$ is annihilated by $Q^{\prime}$, then $Q^{\prime} \in \operatorname{class}(Q)=$ class $(P)$ by Lemma 3 , hence $c \in \mathscr{C}(S) \subset \mathscr{C}\left(Q^{\prime}\right)$, hence $c$ operates regularly on the nonsingular $R / Q^{\prime}$-module $\overline{e R}$; a contradiction to $e c=0$. Thus and by left-right-symmetry, $S$ is localizable hence classical, hence a union of clans ([16], Theorem 4); in particular $P$ belongs to a clan.

Assume conversely that $P$ belongs to a clan; put $S=\bigcap$ clan $(P)$ and select $P^{\prime} \in \operatorname{clan}(P)$ with maximal $\kappa\left(R / P^{\prime}\right)=\alpha$. If $P^{\prime} m \rightarrow \rightarrow$ then by Lemma 3 there is $e \in E_{P^{\prime}}$ with ann $(e R) \subset Q$; by [8], 4.4(5) there is $n$ with $e S^{n}=0$. Thus $S^{n} \subset \operatorname{ann}(e R) \subset Q$ hence there is $P^{\prime \prime} \in \operatorname{clan}(P)$ with $P^{\prime \prime} \subset Q$. Consequently

$$
\kappa\left(R / P^{\prime \prime}\right) \geqq \kappa(R / Q)=\kappa\left(R / P^{\prime}\right)=\alpha ;
$$

and by maximality of $\alpha, \kappa\left(R / P^{\prime \prime}\right)=\alpha$ hence $Q=P^{\prime \prime} \in \operatorname{clan}(P)$. This shows class $\left(P^{\prime}\right) \subset \operatorname{clan}(P)$; hence class $\left(P^{\prime}\right)$ is finite and therefore by the preceeding consideration classical; therefore class $\left(P^{\prime}\right)=\operatorname{clan}(P)$.

COROLlaRY 6. Every clan of an FBN-ring is contained in a stratum.

If $\bar{R}$ is a factorring of $R$, then a link $\bar{P} m \bar{Q}$ in $\bar{R}$ gives rise to a link $P \leadsto Q$ in $R$, but not necessarily vice versa: we write $\bar{R}$-class $(P) \leadsto \bar{R}$-class $(Q)$ if there exist $P^{\prime} \in \bar{R}$-class $(P)$ and $Q^{\prime} \in \bar{R}$-class $(Q)$ with $P^{\prime} \rightsquigarrow Q^{\prime}$. 
THEOREM 7. Let $R$ be an FBN-ring with prime radical $N$, let $\bar{R}=R / N$, and let $P, Q$, be distinct prime ideals in the $\alpha$-stratum of spec $R$. Then $\bar{R}$-class $(P) \leadsto \bar{R}$-class $(Q)$, if and only if $\bar{R}$-class $(P)=$ $\bar{R}$-class $(Q)$ or there exist $P^{\prime \prime} \in \bar{R}$-class $(P)$ and $Q^{\prime \prime} \in \bar{R}$-class $(Q)$ such that $\mathrm{rt}_{-\mathrm{ann}_{R}}\left(N / Q^{\prime \prime} N\right) \subset P^{\prime \prime}$.

Proof. By Proposition 2 (4), the links in $R$ and $R / N^{2}$ are the same; hence assume without loss of generality $N^{2}=0$.

1. $\bar{R}=R / N$ is again an FBN-ring, and the indecomposable injective $\bar{R}$-module corresponding to the prime ideal $\bar{P}$ is $U_{P}=$ $\operatorname{ann}_{E_{P}}(N)$. The exact sequence $0 \rightarrow N \rightarrow R \rightarrow \bar{R} \rightarrow 0$ yields under the functor $\operatorname{hom}_{R}\left(-, E_{P}\right)$ the exact sequence $0 \rightarrow U_{P} \rightarrow E_{P} \rightarrow H_{P} \rightarrow 0$ where $H_{P}=\operatorname{hom}_{R}\left(N, E_{P}\right)=\operatorname{hom}_{\bar{R}}\left(N, U_{P}\right)$, using $N^{2}=0$. The injective hull of $H_{P}$ as $\bar{R}$-module is the direct sum of $\alpha_{i}$-smooth indecomposable injective $U_{Q_{i}}$, since $\vec{R}$ is FBN; as $H_{P} \cap U_{Q_{i}} \neq 0$ by essentiality and as $H_{P}$ is a factor of the $\alpha$-smooth module $E_{P}, \alpha_{i} \leqq \alpha$.

A given link $P m Q$ yields a nonzero homomorphism $\phi: E_{P} \rightarrow E_{Q}$ by Proposition 4. $\phi$ has either nonzero restriction $U_{P} \rightarrow U_{Q}$, in which case $\bar{P} \leadsto m \bar{Q}$ in $\bar{R}$, i.e., $\bar{R}$-class $(P)=\bar{R}$ class $(Q)$; or zero restriction to $U_{P}$, in which case it induces a nonzero homomorphism $\bar{\phi}: H_{P} \rightarrow U_{Q}$. Then $\bar{\phi}$ extends to the $\bar{R}$-injective hull $\bigoplus U_{Q_{i}}$ of $H_{P}$, and some component $\phi_{i}: U_{Q_{i}} \rightarrow U_{Q}$ of this extension is nonzero. For such $i$, $\alpha_{\imath} \geqq \kappa(\bar{R} / \bar{Q})=\alpha$ hence $\alpha_{i}=\alpha$, hence $\bar{Q}_{i} m m \bar{Q}$ or $Q_{i} \in \bar{R}$-class $(Q)$.

By essentiality there exists $0 \neq \beta \in H_{P} \cap U_{Q_{i}}$ with $\beta Q_{i}=0$; this means $\beta\left(Q_{i} N\right)=0$ hence induces $0 \neq \bar{\beta}: N / Q_{2} N \rightarrow U_{P}$. Consequently the right- $\bar{R}$-module $N / Q_{i} N$ is not $\bar{P}$-torsion, hence the ideal rt-ann $\left(N / Q_{i} N\right)$ is contained in $P$. This proves one direction of the theorem.

2. Assume conversley rt-ann $(N / Q N) \subset P$. Then the right-module $N / Q N$ cannot be $\bar{P}$-torsion: otherwise and since it is finitely generated as left-module, rt-ann $(N / Q N)$ would be $\bar{P}$-dense hence $\not \subset P$. Consequently there exists $0 \neq \beta: N / Q N \rightarrow U_{P}$; and we may choose $\beta R$ uniform; notice $\beta Q=0$. Then for any $0 \neq \gamma \in \beta R$ we have $B=$ ann $(\gamma R) \supset Q ; \quad$ and since $\alpha=\kappa(R / Q) \geqq \kappa(R / B) \geqq \mathrm{lt}-\kappa(N / B N)$, which equals by [9], $2.2 \mathrm{rt}-\kappa(N / B N) \geqq \kappa(N / \operatorname{ker} \gamma)$, which equals $\alpha$ since $N /$ ker $\gamma$ embedds into the $\alpha$-smooth module $U_{P}$, one gets $\alpha=\kappa(R / B)$ hence $B=Q$ by [5], 7.5 and 7.2. This shows that $\beta R$ is a uniform prime hence $\alpha$-criterical module ([9], 2.5).

Pick $e \in E_{P}$ which maps to $\beta \in H_{P}$ under the surjection $E_{P} \rightarrow H_{P}$, 
and take an $\alpha$-composition series of $e R \cap U_{P}$, all whose factors have annihilators in $\bar{R}$-class $(P)$ by Lemma 3 , applied to the $\bar{R}$-module $U_{P}$. As $\beta R \cong e R /\left(e R \cap U_{P}\right)$ is $\alpha$-critical with annihilator $Q$, one has an $\alpha$-composition series of $e R$ whose top factor has annihilator $Q$ while all other factors have annihilators in $\bar{R}$-class $(P)$.

Now take among all $\alpha$-composition series of finitely generated submodules of $E_{P}$ with these properties, one of shortest length, say $0=B_{0} \varsubsetneqq \cdots \varsubsetneqq B_{n}$. As in the proof of Proposition 4,

$$
0 \longrightarrow B_{n-1} / B_{n-2} \longrightarrow B_{n} / B_{n-2} \longrightarrow B_{n} / B_{n-1} \longrightarrow 0
$$

yields a link $\bar{R}$-class $(P) \ni \operatorname{ann}\left(B_{n-1} / B_{n-2}\right) \rightsquigarrow \operatorname{ann}\left(B_{n} / B_{n-1}\right)=Q$ hence $\bar{R}$-class $(P) m \bar{R}$-class $(Q)$, as desired.

3. It remains to deduce the same conclusion from the assumption $\bar{R}$-class $(P)=\bar{R}$-class $(Q)$. But then $\bar{R}$-class $(P)$ has at least the two members $P$ and $Q$, and then a link $\overline{P^{\prime}} m \overline{P^{\prime \prime}}$ between suitable $P^{\prime}$, $P^{\prime \prime} \in \bar{R}$-class $(P)$ obviously exists.

REMARKS. (1) Note that the criterion of Theorem 7 really involves only $R / N$-modules: rt-ann $\overline{\bar{R}}\left(N / Q^{\prime \prime} N\right) \subset \bar{P}$.

(2) If rt-ann $_{R}(N / Q N) \subset P$ and $\kappa(R / P)=\kappa(R / Q)$, then

$$
\begin{aligned}
\kappa(R / Q) & \geqq \kappa(R / \mathrm{lt} \text {-ann }(N / Q N))=\mathrm{It}-\kappa(N / Q N)=\operatorname{rt}-\kappa(N / Q N) \\
& =\kappa(R / \mathrm{rt}-\operatorname{ann}(N / Q N)) \geqq \kappa(R / P)
\end{aligned}
$$

by [9], 2.1 and 2.2; hence equality holds throughout. The first equality implies $Q=\operatorname{lt}$-ann $(N / Q N)$, and the second one that $P$ is a minimal prime ideal over rt-ann $(N / Q N)$.

Hence for given $Q$, such prime ideals $P$ exist if and only if lt-ann $(N / Q N)=Q$, ann in this case there are only finitely many of them (since a noetherian ring has only finitely many minimal prime ideals). This means that any $Q$ is directly linked in $R$ to only finitely many $\bar{R}$-classes; consequently if $\mid \bar{R}$-class $(P) \mid \leqq \preccurlyeq$ for some infinite cardinal $\aleph$ and all $P$, then also $\mid R$-class $(P) \mid \leqq \aleph$.

III. Noetherian PI-rings. Let $R$ be a noetherian PI-ring, i.e., a noetherian ring with a polynomial identity with integer coefficients which is proper on every nonzero factorring [18]. For a prime ideal $Q$ of $R$, PI-degree $(Q)=n$ if $R / Q$ satisfies $S_{2 n}$ but not $S_{2 n-2}$. PIdegree $(R)=\max \{\mathrm{PI}$-degree $(Q): Q \in \operatorname{spec} R\}$ is a well defined natural number, for any PI-ring $R$.

If $R$ is a semiprime PI-ring and if $Q$ is a prime ideal of $R$ with 
PI-degree $(Q)=$ PI-degree $(R)=n$, then $Q$ doesn't contain all evaluations of the Formanek polynomial [4] for $n \times n$-matrices, hence the commutative localization $R_{\Pi}$ at the prime ideal $\Pi=Q \cap$ centre $(R)$ of the centre inverts all elements of $\mathscr{C}(P)$ ([2], [21], [18]); thus such $Q$ is classical, i.e. $\{Q\}$ is a clan.

Define $N_{n}=\bigcap\{Q \in \operatorname{spec} R$ : PI-degree $(Q) \leqq n\}$, a semiprime ideal of the noetherian PI-ring $R$. For any factorring $R / I$ with $I \subset N_{n}$ which has only prime ideals of PI-degree $\leqq n$, in particular for $R /\left(N_{n}^{2}+N_{n+1}\right)$, the $R / I$-classes are described by Theorem 7 via the $R / N_{n}$-bimodules $N_{n} /\left(Q N_{n}+N_{n+1}\right)$ and $N_{n} /\left(N_{n} Q+N_{n+1}\right)$ as certain mergers of $R / N_{n}$-classes. On the other hand $R / N_{n+1}$-class $(P)=\{P\}$ if PI-degree $(P)=n+1$ by the preceding paragraph, and $R / N_{n+1^{-}}$ class $(Q)=R /\left(N_{n}^{2}+N_{n+1}\right)$-class $(Q)$ if PI-degree $(Q) \leqq n$, since any link $\bar{Q} \leadsto \bar{Q}^{\prime}$ between prime ideals of $R / N_{n+1}$ of PI-degree $\leqq n$ shows already in $R /\left(Q^{\prime} Q+N_{n+1}\right)$ hence a fortiori in $R /\left(N_{n}^{2}+N_{n+1}\right)$; cf. Proposition 2.

Summarizing: in passing from $R / N_{n}$ to $R /\left(N_{n}^{2}+N_{n+1}\right)$ (or to $R$ if PI-degree $(R)=n$ ) certain $R / N_{n}$-classes merge to form the $R /\left(N_{n}^{2}+N_{n+1}\right)$-classes (or $R$-classes) according to Theorem 7 ; in passing from $R /\left(N_{n}^{2}+N_{n+1}\right)$ to $R / N_{n+1}$ certain new singleton $R / N_{n+1}$-classes appear but no mergers occur.

Climbing in this way from the commutative ring $R / N_{1}$ in finitely many steps to the noetherian PI-ring $R$, provides a description of its classes. Combining this with Remark (2) after Theorem 7 yields:

CoROllary 8. For every noetherian PI-ring, the classes are at most countable.

Unfortunately the step from countable to finite classes, i.e., clans, is still difficult; we have only a rather trivial positive result and a couple of counterexamples:

Proposition 9. If an FBN-ring satisfies INC with respect to its centre, then it has enough clans. In particular this applies to any noetherian ring which is integral over its centre, and to every prime noetherian PI-ring of Krull dimension one.

Proof. For an FBN-ring with centre $C$, the intersection $Q \cap C$ is the same for all $Q \in \operatorname{class}(P)$ : if $a \in Q \cap C$ and $P \leadsto Q$, then by Proposition 2 (4) for the ideal $I=a R, \quad(Q \cap P) I=a(Q \cap P) \subset Q P$ hence $a \in a R \subset P$. Therefore if $R$ satisfies INC (i.e., if prime ideals of $R$ over the same ideal of $C$ are incomparable [10]), then all $Q \in \operatorname{class}(P)$ are minimal over $(P \cap C) R$ hence finite in number; so 
Theorem 5 yields the result.

If $R$ is noetherian and integral over $C$, it is automatically fully bounded [20] and has INC [6]. If $R$ is a prime noetherian PI-ring: of Krull dimension one, all nonzero prime ideals are maximal and have nonzero intersection with $C$ [17], hence INC holds again.

REMARKs. (1) Every FBN-ring of Krull dimension zero has enough clans, in fact is artinian. We do not know whether every prime FBN-ring of Krull dimension one has enough clans; apart from our result for noetherian PI-rings this is true for HNP-rings (cf. [16]), by a proof in the spirit of our approach [12].

(2) Our argument also shows the following: for any prime ideal $\Pi$ of the centre $\mathrm{C}$ of any FBN-ring $R$, the prime ideals $P$ of $R$ with maximal $\kappa(R / P)$ among those containing $\Pi$, belong to clans. Note that such $P$ exist for every $\Pi$.

CountereXAMPle 1. A noetherian PI-ring of Krull dimension one with infinite classes.

The example. Put $R=A \times A_{\sigma}$, the split extension of a commutative noetherian domain $A$ by the bimodule $A_{\sigma}$, with the right$A$-module structure modified by an automorphism $\sigma$ of $A$.

$A_{\sigma}$ is an ideal of $R$ of square zero, hence $R$ satisfies $S_{2}^{2}$ and has prime radical $N=A_{\sigma}$. There is a one-to-one correspondence between the prime ideals $P$ of $A$ and of $R$; we do not distinguish between them notationally. One has $\kappa(R)=\kappa(A)$; and $N / P N=A_{\sigma} / P A_{\sigma}=(A / P)_{\sigma}$, hence lt-ann $(N / P N)=P$ and rt-ann $(N / P N)=\sigma^{-1}(P)$; therefore by Theorem 7 , class $(P)=\left\{\sigma^{n}(P): n \in Z\right\}$.

It is easy to find instances where $\kappa(A)=1$ and class $(P)$ is infinite; e.g. let $A=K[x]$ for a field $K$ of characteristic zero, $\sigma(x)=x+1$ and $P=\langle x\rangle$ (this example is mentioned in [16]).

Variations. $A=K[x, y]_{\langle x, y\rangle}$ and $\sigma(x)=x+y, \sigma(y)=y$ produces a local noetherian PI-ring $R$ of Krull dimension two, with infinite classes in the middle stratum of spec $R$. The case of a proper endomorphism $\sigma$ can also be discussed.

CountereXAMPLe 2. A prime noetherian PI-ring of Krull dimension two with infinite classes.

A general construction. Let $C$ be a commutative noetherian domain, with two noetherian subrings $A$ and $B$, both containing the nonzero semiprime principal ideal $I=c C$ of $C$; consider the non- 
commutative ring $R=\left(\begin{array}{ll}A & I \\ I & B\end{array}\right) . \quad R$ is prime, noetherian, and satisfies all identities of $2 \times 2$-matrices over $\boldsymbol{Z}$. Its Krull dimension equals the maximum of the Krull dimensions of $A$ and $B$. The centre is $A \cap B$, embedded diagonally; it is not necessarily noetherian. The Formanek centre, i.e. the collection of all evaluations of central polynomials with zero constant term for $M_{2}(Z)$, equals $I^{2}$ (use the central polynomial $\left.[x, y]^{2}=-\operatorname{det}([x, y])\right)$.

A prime ideal of $R$ of PI-degree two doesn't contain the Formanek centre, hence doesn't contain $I$. By [18], 4.16(c) these prime ideals are in one-to-one correspondence with the prime ideals of the centre $A \cap B$ not containing $I$; they constitute singleton clans.

The prime ideals of $R$ of PI-degree one contain the Formanek centre hence $I$; explicitely there are two types $\mathscr{P}=\left(\begin{array}{ll}P & I \\ I & B\end{array}\right)$ and $\mathbb{Q}=\left(\begin{array}{ll}A & I \\ I & Q\end{array}\right)$ for arbitrary prime ideals $P$ and $Q$ containing $I$, of $A$ and $B$ respectively. The intersection of all these prime ideals is $N=N_{1}=M_{2}(I) ;$ to apply Theorem 7 one computes

$$
N / \mathscr{Q} N=\left(\begin{array}{ll}
I / I & I / I \\
I / Q I & I / Q I
\end{array}\right), \quad \operatorname{lt} \text {-ann }(N / \mathscr{Q} N)=\left(\begin{array}{ll}
A & I \\
I & B \cap Q C
\end{array}\right)
$$

and rt-ann $(N / \mathscr{Q} N)=\left(\begin{array}{l}A \cap Q C \\ I\end{array} \quad \begin{array}{l}I \\ B\end{array}, Q C\right)$, using at this point $I=c C$ and cancelling $c$. By Theorem 7 and the remarks following it, a link into $\mathscr{Q}$ exists if and only if $B \cap Q C=Q$, and in this case there is precisely a self-link $\mathscr{Q} \leadsto \mathbb{Q}$ and at least one, at most finitely many links $\mathscr{P} m \mathscr{Q}$, namely for the $\mathscr{P}=\left(\begin{array}{ll}P & I \\ I & B\end{array}\right)$ with $P \supset A \cap Q C$ and $\kappa(A / P)=\kappa(B / Q)$.

By symmetry (transposition is an antiautomorphism of $R$ ) $\mathscr{P} \leadsto \mathscr{Q}$ implies $\mathscr{Q} \leadsto \mathscr{P}$ and vice versa. One may therefore, ignoring direction and self-links and passing to factors modulo $I$, represent all links by a bipartite locally finite graph between spec $\bar{A}$ and spec $\bar{B}$, where the existence of an edge is given by $\kappa(\bar{A} / \bar{P})=\kappa(\bar{B} / \bar{Q})$ and $\bar{P} \supset \bar{A} \cap \overline{Q C}$ (or equivalently $\bar{Q} \supset \bar{B} \cap \overline{P C}$ ).

The example (appears in [19] in a different context). Let $k=\boldsymbol{Q}(\sqrt{6}), K=\boldsymbol{Q}(\sqrt{2}, \sqrt{3})=k(a)$ where $a=\sqrt{3}-\sqrt{2}$, and let $a^{*}=-a$ be the nontrivial automorphism of $K / k$. Let $C=K[x, y]$, $A=k[x+\sqrt{3}, y, \sqrt{3} y], \quad B=k[x+\sqrt{2}, y, \sqrt{2} y] \quad$ and $\quad I=y C$. Then $R$ has Krull dimension two as desired; the two nonmaximal 
PI-degree-one prime ideals $\left(\begin{array}{ll}A & I \\ I & I\end{array}\right)$ and $\left(\begin{array}{ll}I & I \\ I & B\end{array}\right)$ form a clan; but the maximal PI-degree-one ideals determine infinite classes.

Indeed consider the class determined by the prime ideal of $R$ which in turn is determined by the irreducible polynomial $q$ of $\bar{B}=k[x+\sqrt{2}]$, with roots $\rho_{1}, \cdots, \rho_{s}$ in the algebraic closure of $k$. For $t=x+\sqrt{3}, q(t-a) q(t+a) \in \bar{A} \cap \overline{Q C}$ since it is invariant under the automorphism *; conversely if $f(t)$ generates the $\bar{A}$-ideal $\bar{A} \cap \overline{Q C}$, then $f(t)=q(t-a) g(t)$ for some $g \in \bar{C}$ hence $f(t)=f(t)^{*}=$ $g(t+a) g^{*}(t)$ hence $f(t)^{2}=g(t-a) q(t+a) q(t) g^{*}(t)$ with $g g^{*} \in \bar{A}$; therefore the roots of $f(t)$ are precisely $\rho_{i} \pm a$.

Consequently the prime ideals $\mathscr{P}$ and $\mathscr{Q}$ in the class in question, correspond to the minimal polynomials of all the $\rho_{i}+n a$ for even respectively odd integers $n$; since these are infinite in number, the class contains infinitely many prime ideals of both types.

Variations. With the choice of $C, A, B$ and $I$ made above, $\left(\begin{array}{ll}A / I^{2} & I / I^{2} \\ I / I^{2} & B / I^{2}\end{array}\right)$ and $\left(\begin{array}{cc}A / I^{2} & I / I^{2} \\ 0 & B / I^{2}\end{array}\right)$ have also infinite classes and Krull dimension one (as does counterexample 1), and they satisfy all identities of $2 \times 2$-matrices over $Z$.

For the choice (cf. [19]) $C=F[x], A=F_{1}+x F[x], B=F_{2}+x F[x]$, $I=x F[x]$ for finite-dimensional field extensions $F / F_{i}$, one has $\kappa(R)=\kappa(A)=\kappa(B)=1$; hence $R$ has enough clans by Proposition 9 . $F /\left(F_{1} \cap F_{2}\right)$ may be chosen finite-dimensional, algebraic or transcendental; then $R$ is finitely generated as module, integral or nonintegral over its centre $\left(F_{1} \cap F_{2}\right)+x F[x]$, respectively.

\section{REFERENCES}

1. H. Cartan and S. Eilenberg, Homological Algebra, Princeton Univ. Press, 1956.

2. A. W. Chatters, Localization in P. I. rings, J. London Math. Soc., 2 (1970), 763-768.

3. D. Eisenbud and J. C. Robson, Hereditary noetherian prime rings, J. Algebra 16 (1970), 86-104.

4. E. Formanek, Central polynomials for matrix rings, J. Algebra, 23 (1972), 129-132.

5. R. Gordon and J. C. Robson, Krull dimension, Memoirs Amer. Math. Soc., 133 (1973).

6. A. G. Heinicke, A remark about noncommutative integral extensions, Canad. Math. Bull., 13 (1970), 359-361.

7. A. V. Jategaonkar, The torsion theory at a semiprime ideal, A. Acad. Brasil. Cienc., 45 (1973), 197-200.

8. A. V. Jategaonkar, Injective modules and localization in non-commutative noetherian rings, Trans. Amer. Math. Soc., 188 (1974), 109-123.

9. A. V. Jategaonkar, Jacobson's conjecture and modules over fully bounded noetherian rings, J. Algebra, 30 (1974), 103-121. 
10. I. Kaplansky, Commutative Rings, Univ. of Chicago Press, 1974.

11. G. Krause, On fully left-bounded left-noetherian rings, J. Algebra, 23 (1972), 88-99. 12. T. H. Lenagan, Bounded hereditary noetherian prime rings, J. London Math. Soc., 6 (1973), 241-246.

13. G. Michler, Right symbolic powers and classical localization in right noetherian rings, Math. Z., 127 (1972), 57-69.

14. B. J. Müller, On semi-perfect rings, Illinois J. Math., 14 (1970), 464-467.

15. - The structure of quasi-Frobenius rings, Canad. J. Math., 26 (1974), $1141-1151$.

16. Localization in non-commutative noetherian rings, Canad. J. Math., 28 (1976), 600-610.

17. L. Rowen, Some results on the center of a ring with polynomial identity, Bull. Amer. Math. Soc., 79 (1973), 219-223.

18. - On rings with central polynomials, J. Algebra, 31 (1974), 393-426.

19. W. Schelter, Integral extensions of rings satisfying a polynomial identity, preprint.

20. J. Shapiro, A noncommutative analog to prime ideals, Ph. D. thesis Rutgers Univ. 1975.

21. L. Small, Localization in P.I.-rings, J. Algebra, 18 (1971), 269-270.

Received December 24, 1975 and in revised form July 6, 1976. Supported in part by the National Research Council of Canada.

McMaster University 



\section{PACIFIC JOURNAL OF MATHEMATICS}

EDITORS

RICHARD ARENS (Managing Editor)

University of California

Los Angeles, California 90024

R. A. BEAUMONT

University of Washington

Seattle, Washington 98105

J. DugundjI

Department of Mathematics

University of Southern California

Los Angeles, California 90007

D. Gilbarg and J. Milgram

Stanford University

Stanford, California 94305

\section{ASSOCIATE EDITORS}

E. F. BeCKENBACH

B. H. NeUMaNN

F. WOLF

K. YoSHIDA

\section{SUPPORTING INSTITUTIONS}

UNIVERSITY OF BRITISH COLUMBIA

CALIFORNIA INSTITUTE OF TECHNOLOGY

UNIVERSITY OF CALIFORNIA

MONTANA STATE UNIVERSITY

UNIVERSITY OF NEVADA

NEW MEXICO STATE UNIVERSITY

OREGON STATE UNIVERSITY

UNIVERSITY OF OREGON

OSAKA UNIVERSITY

\author{
UNIVERSITY OF SOUTHERN CALIFORNIA \\ STANFORD UNIVERSITY \\ UNIVERSITY OF TOKYO \\ UNIVERSITY OF UTAH \\ WASHINGTON STATE UNIVERSITY \\ UNIVERSITY OF WASHINGTON \\ AMERICAN MATHEMATICAL SOCIETY \\ NAVAL WEAPONS CENTER
}




\section{Pacific Journal of Mathematics \\ Vol. 67, No. $1 \quad$ January, 1976}

Gregory Wayne Brumfiel and John W. Morgan, Homotopy theoretic

consequences of $N$. Levitt's obstruction theory to transversality for

spherical fibrations . ................................ 1

Jacob Burbea, Total positivity of certain reproducing kernels ........... 101

Wai-Mee Ching, The structure of standard $C^{*}$-algebras and their

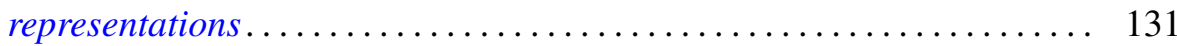

Satya Deo, The cohomological dimension of an $n$-manifold is $n+1 \ldots \ldots 155$

Masahiko Fujiwara and Masaki Sudo, Some forms of odd degree for which the Hasse principle fails ................................. 161

Mikihiro Hayashi, Smoothness of analytic functions at boundary points ... 171

Rebecca A. Herb, A uniqueness theorem for tempered invariant

eigendistributions ................................ 203

David Alan Legg, Orlicz space convergence of martingales of

Radon-Nikodým derivatives given a $\sigma$-lattice.................. 209

D. B. McAlister, v-prehomomorphisms on inverse semigroups......... 215

Bruno J. Mueller, Localization in fully bounded Noetherian rings . ........ 233

Donald J. Newman and A. R. Reddy, Rational approximation to $x^{n} \ldots \ldots .247$

Abraham Ziv, Inclusion relations between power methods of limitation.... 251 